\title{
Does the Combined Use of Magnification Loupes and Harmonic FOCUS Improve the Outcome of Thyroid Surgery?
}

\author{
Luca P Suffat ${ }^{1}$, Eugenia Lavorini ${ }^{2}$, Guido Mondini ${ }^{3}$, Ivan Lettini ${ }^{4}$, Giulia Osella ${ }^{5}$, Lodovico Rosato ${ }^{6}$
}

\begin{abstract}
Introduction: Blood loss, injury of recurrent laryngeal nerves, and damage of the parathyroid glands are the most dangerous complications during total thyroidectomy. The aim of our study is to compare the combined use of optical magnification and ultrasound hemostatic devices with traditional surgical suture ligation in terms of operating time, hospital stay, and surgical complications as bleeding, hypoparathyroidism, and recurrent laryngeal nerve injuries.

Materials and methods: A total of 140 patients undergoing total thyroidectomy for either diffuse or nodular goiter were enrolled between January 2016 and June 2019 at our endocrine surgery unit. A total of 70 patients operated with magnifying loupes and ultrasonic scalpel, defined as group A, were compared with other 70 patients operated without loupes and with the aid of conventional hemostatic devices, named as group B. Results: Demographics were comparable between the assessed groups. Operating time was longer in group $B$ (mean length of 110 minutes vs 79 minutes, $p<0.01$ ). Intraoperative blood loss was lower in group A ( $22 \mathrm{~mL}$ vs $38 \mathrm{~mL}, p<0.01$ ). There were no differences between both groups in terms of postoperative bleeding, hematoma formation, pain control, and length of stay. Transient recurrent laryngeal nerve damage was observed in one case in group A and two cases in group B. Transient hypocalcemia secondary to hypoparathyroidism was more common in group $B$ than in group $A$ ( 31 patients vs 27 patients).

Conclusion: In our study, use of magnification loupes and harmonic scalpel is associated with significant reduction of operating/anesthesia time and intraoperative blood loss.

Clinical significance: Our results and suggestions can improve the standardized use of magnification loupes and harmonic scalpel in thyroid surgery.

Keywords: Harmonic scalpel, Magnification loupes, Post-thyroidectomy complications, Thyroid surgery.

World Journal of Endocrine Surgery (2020): 10.5005/jp-journals-10002-1283
\end{abstract}

\section{INTRODUCTION}

Total thyroidectomy and lobohystmectomy are the most common procedures in endocrine surgery. ${ }^{1}$ According to the Italian Health Department, 32,668 patients underwent thyroidectomy in 2017 in Italy for benign and malignant disease.

In the late nineteenth century, thyroidectomy was a highly morbid procedure as it was associated with a potential massive blood loss and a mortality rate of about 50\%. Emil Theodor Kocher (1841-1917) revolutionized thyroid surgery with the introduction of the total extracapsular thyroidectomy with suture ligation of the major arteries. This innovative technique resulted in decreasing the mortality rate from 40 to $0.2 \%$, as reported by CAT Billroth in $1895 .{ }^{2}$ Despite postoperative bleeding can be a devastating complication of thyroid surgery, the most crucial steps of the surgical procedure are the identification and preservation of the recurrent laryngeal nerves (RLNs) and parathyroid glands. ${ }^{3}$

Thus, there are three key points in the surgical technique: ligation of all blood vessels as close as possible to the thyroid capsule, identification and preservation of RLNs, as well as of parathyroid glands and their vascularization.

Moreover, a shorter operating time, without increased complications, could lead to optimize the use of operating room resources and to avoid a long-duration general anesthesia.

In this study, we evaluated the impact of optical magnification (loupes) and ultrasound hemostatic devices, with the aim of highlighting any benefit in terms of surgical procedures, operating times, and outcome in thyroid surgery. ${ }^{4}$

\footnotetext{
${ }^{1-6}$ Department of Surgery and Oncology ASL TO4, Endocrine Surgical Unit and General Surgery, iVREA, Italy

Corresponding Author: Giulia Osella, Department of Surgery and Oncology ASL TO4, Endocrine Surgical Unit and General Surgery, iVREA, Italy, Phone: +39 0125414243, e-mail: giulia.osella89@gmail. com

How to cite this article: Suffat LP, Lavorini E, Mondini G, et al. Does the Combined Use of Magnification Loupes and Harmonic FOCUS Improve the Outcome of Thyroid Surgery? World J Endoc Surg 2020;12(1):18-22.

Source of support: Nil

Conflict of interest: None
}

\section{Materials and Methods}

\section{Patients}

We performed a retrospective study that included 140 patients diagnosed with either diffuse or nodular goiter and surgically treated with total thyroidectomy between January 2016 and June 2019 at our endocrine surgery unit.

A complete preoperative assessment was carried out on all patients, including a complete blood count, thyroid function tests, and ultrasound examination. Fine-needle aspiration cytology was performed for all goiters with nodules $>1 \mathrm{~cm}$, which appeared suspect or dubious after ultrasound (US) in order to exclude malignancy. All patients underwent a preoperative laryngoscopy to assess vocal cords mobility.

The patients were divided into two groups: group A was composed of patients who underwent total thyroidectomy with 
Table 1: Demographic parameters and nature of the disease

\begin{tabular}{llll}
\hline & \multicolumn{2}{c}{ Group $A$} & \multicolumn{2}{c}{ Group $B$} & \\
\cline { 2 - 3 } Parameter & $n=70$ & $n=70$ & p value \\
\hline Females $(n, \%)$ & $49(70.0 \%)$ & $52(74.3 \%)$ & 0.413 \\
Age (mean \pm SD, years) & $52 \pm 12.1$ & $54 \pm 12.1$ & 0.893 \\
Benign nature $(n, \%)$ & $65(92.9)$ & $66(94.3)$ & 0.600 \\
\hline
\end{tabular}

the device of ultrasonic scalpel and with $2.5 \times$ magnifying loupes, used only by the lead surgeon; group $B$ consisted of patients who underwent conventional surgery with the use of clamp and tie technique and electrocautery method for hemostasis and dissection.

Group A included 70 patients, 49 females and 21 males, with a mean age of 52 years (range 21-64); group B was composed of 70 patients, 52 females and 18 males, with a mean age of 54 years (range 23-67). These results are shown in Table 1.

For all patients, surgery was first suggested by the endocrinologist, then confirmed by the endocrine surgeon. The diseases that determined the need for surgery were the following:

- Group A: 3 Graves' disease (4.3\%), 18 toxic nodular struma (25.7\%), 49 multinodular goiter (70.0\%).

- Group B: 4 Graves' disease (5.7\%), 16 toxic nodular struma (22.9\%), 50 multinodular goiter (71.4\%).

Thyroid volumes measured by ultrasound were comparable in both groups:

- Group A: Males, mean $57.3 \mathrm{~mL}$ (range 24-80); females, mean $50.6 \mathrm{~mL}$ (range 22-68).

- Group B: Males, mean $58.1 \mathrm{~mL}$ (range 26-76); females, mean $51.8 \mathrm{~mL}$ (range 20-69).

Clinical presentation and patient characteristics were similar and comparable in both groups.

\section{Exclusion Criteria}

The sample population excluded patients with the following characteristics:

- Those with preoperative findings of thyroid neoplasia

- Those who needed a central or lateral compartment lymphadenectomy for oncological reason

- Those with recurrent goiter

- Those with previous neck irradiation and radioiodine ablation

\section{Surgery}

The lead surgeon, as well as the whole surgical team, was the same for all patients, and all had proven experience in endocrine surgery. Out of a population of patients with the characteristics identified as relevant for this study, the patients chosen for the specific sample population were the last 70 patients operated with conventional surgery (group B) and the first 70 operated with the routine use of the ultrasonic scalpel device and $2.5 \times$ magnifying loupes (group A).

In both groups, all procedures were carried out under general anesthesia and using the same standardized surgical technique:

- Patient positioned in the supine position with neck slightly hyperextended and arms abducted
- Minimal cervicotomy incision, according to the goiter volume, was performed, detaching the upper flap up to the thyroid cartilage and the lower flap down to the jugulum

- Incision of the alba-colli line with interruption of the prethyroid muscles, only if necessary and limited to the thyroid insertion of the sternothyroid muscle

- Identification of the cervical vascular bundle

- Ligation of the superior thyroid artery. Special care must be exercised in saving the external branch of the superior laryngeal nerve, which only rarely can be identified

- Identification of the RLNs laterally in the paratracheal groove from their origin in the mediastinum up to the fibers of the inferior pharyngeal constrictor muscle

- Identification of the parathyroid glands, preserving their anatomical integrity and venous and arterial vascularization

- Special care must be taken to binding a small arterial branch so-called retroneural - which is usually located near the entrance of the RLN under the inferior pharyngeal constrictor muscle

- Conclusion of the procedure with careful control of hemostasis performing the Valsalva maneuver (increasing intrapulmonary pressure up to $40 \mathrm{~cm}$ of $\mathrm{H}_{2} \mathrm{O}$ ) with the aid of the anesthetist

- Layered wound closure, with continuous suturing of the albacolli line, leaving an adequate path in the lower part between the thyroid lodge and the subcutaneous space

- Suction drain, removed after 24-48 hours, before discharge from the hospital.

In group A, harmonic FOCUS was used for dissection and coagulation throughout the entire surgical procedure, except for the superior and inferior thyroid arteries and veins with diameter of $\geq 5 \mathrm{~mm}$, which were ligated and dissected.

In group B, surgical procedures were performed using the conventional clamp-and-tie technique.

The ligatures were performed, always extracapsular, with vicryl $2 / 0$ sutures for the main vascular pedicles and $3 / 0$ for minor vessels, with the aid of bipolar coagulation.

Various intra- and postoperative parameters were assessed and analyzed, including operating time, blood loss, postoperative pain, drainage volume, seroma and hematoma formation, recurrent laryngeal nerve injuries, hypocalcemia secondary to hypoparathyroidism, and length of hospitalization stay.

\section{Statistical Analysis}

Data were expressed as mean \pm standard deviation for continuous variables and as frequency and percentage for categorical variables.

Differences between the two groups were analyzed with the Student's $t$-test for continuous variables and the $\chi^{2}$ test for categorical variables. A $p$ value $<0.05$ was considered as statistically significant. Data were analyzed using the Stata 14 software (Stata Corp., College Station, USA).

\section{Results}

Both groups had similar demographics, diagnosis, volume of goiter, and euthyroidism.

Five patients in group $A$ and four patients in group B were incidentally diagnosed with thyroid cancer, as shown in Table 1. Histologically, all of the cancers were papillary thyroid carcinomas $\leq 1 \mathrm{~cm}$ in diameter. There was no need for reoperation or lymphadenectomy for nodal involvement. 
Table 2: Operative and postoperative data

\begin{tabular}{llll}
\hline & Group $A$ & Group $B$ & \\
\cline { 2 - 3 } Parameter & $n=70$ & $n=70$ & $p$ value \\
\hline Duration of surgery (mean [min-max] \pm SD, minutes) & $79[67-122] \pm 20.9$ & $110[93-180] \pm 26.3$ & $p<0.001$ \\
Intraoperative blood loss (mean \pm SD, $\mathrm{mL}$ ) & $22 \pm 10$ & $38 \pm 12$ & $p<0.001$ \\
Drainage volume ( mean $\pm \mathrm{SD}, \mathrm{mL})$ & $38 \pm 25.3$ & $60 \pm 30.1$ & $p<0.001$ \\
Length of hospitalization stay (mean $\pm \mathrm{SD}$, hours) & $55 \pm 20.3$ & $60 \pm 24.1$ & 0.187 \\
VAS score (mean $\pm \mathrm{SD}$ ) & $3 \pm 0.8$ & $3 \pm 0.7$ & 0.999 \\
\hline
\end{tabular}

Table 3: Postoperative surgical complications

\begin{tabular}{lccc}
\hline & Group A & Group B & \\
\cline { 2 - 3 } Parameter & $n=70$ & $n=70$ & p value \\
\hline $\begin{array}{l}\text { Paralysis of single RLN } \\
(n, \%)\end{array}$ & $1(1.4 \%)$ & $2(2.9 \%)$ & 0.872 \\
$\begin{array}{l}\text { Temporary hypocalcemia } \\
(n, \%)\end{array}$ & $27(38.6 \%)$ & $31(44.3 \%)$ & 0.861 \\
\hline
\end{tabular}

Operating time was recorded as the time interval between skin incision and wound closure and it was significantly longer in group $B$, with a mean length of 79 minutes (range 67-122 minutes) in group $A$ and 110 minutes (range 93-180 minutes, $p<0.01$ ) in group $B$.

Intraoperative blood loss was significantly lower for group $A$ compared to group $B(22 \mathrm{~mL}$ as against $38 \mathrm{~mL}, p<0.01)$. Subsequent postoperative drainage volumes were significantly decreased in group A.

There was no statistically significant difference between the two groups with respect to the length of hospitalization stay, even though it was slightly shorter in group A (55 hours vs 60 hours).

Postoperative pain, assessed by the VAS score, was mild and showed overlapping results in both groups in the first 24 hours, with a good response to conventional pharmacological pain therapy. These results are shown in Table 2.

The main intraoperative complications were assessed for both groups and are listed in Table 3.

Temporary single RLN palsy occurred in only one patient in group $A$ and in two patients in group B. In all cases, the injury affected the right RLN, as confirmed by a postoperative laryngoscopic examination. The RLN palsy was transient and all patients benefited from a 6 weeks program of logopedic rehabilitation. There were no cases of postoperative bleeding, hematoma, or seroma formation requiring further surgery in either group. No wound infections were recorded in any of the patients involved in this study.

Twenty-seven patients in group A experienced transient and asymptomatic postoperative laboratory hypocalcemia, while 31 patients in group $B$.

All of these patients were discharged with postoperative calcium carbonate and calcitriol supplementation and their serum calcium levels were checked weekly. All the patients were normocalcemic at 30 days; postoperative supplementation was therefore stopped.

\section{Discussion}

The thyroid is a highly vascular organ, with numerous blood vessels entering the parenchyma and approaching recurrent laryngeal nerves and parathyroid glands. Thus, an accurate hemostasis and a delicate dissection are crucial, allowing a good view of the operating field and consequently reducing the risk of injuries of RLNs and of parathyroid glands, as well as intra- and postoperative hemorrhage.

In a conventional thyroidectomy, hemostasis is achieved by traditional clamp, tie, and monopolar or bipolar coagulation., 5, Even if the knot-and-tying technique is quite efficient, it is timeconsuming and it is not associated with a lower risk of bleeding and postoperative hematoma formation. Therefore, over the years many devices have been introduced, in clinical practice, such as clips and numerous topical hemostatic agents. ${ }^{7-9}$

Harmonic FOCUS is an ultrasonic device, introduced in the 1990s, which enables simultaneous cutting and coagulation of medium-caliber vessels with a minimal thermal spread. It is therefore a useful tool in surgical fields where proximity of vital structures does not allow the use of hemostatic thermal devices. ${ }^{10,11}$

The harmonic scalpel delivers mechanical energy in the form of ultrasonic vibrations at a rate of $55 \mathrm{kHz} /$ second. It creates an endovascular coagulum that is capable of sealing off mediumcaliber vessels (up to 5-7 $\mathrm{mm}$ in diameter) and disperses heat within $2 \mathrm{~mm}$ in the surrounding tissue. ${ }^{12,13}$

Many studies showed superior clinical outcome in the use of Harmonic FOCUS compared to the conventional clampand-tie technique in terms of: operative time, postoperative hypoparathyroidism, intraoperative blood loss, and drain output. 11,14

The main concern about the systemic use of energy-based devices in thyroid surgery is their potential thermal insult to the RLNs.

The RLNs preservation is essential in thyroidectomy and their injury is one of the main complications in the surgical procedure. ${ }^{15}$ Recurrent laryngeal nerve injuries often result from inadvertent transection, compression, stretching, electrothermal, ligature entrapment, and ischemia. ${ }^{16}$

Since RLN paralysis could lead to hoarseness and dysphagia, even a transient lesion can cause a reduction of the patient's quality of life.

While the traction nerve injury only disrupts the outer layer of the nerve, thermal injury damages the inner endoneurium. This is more likely to result in a permanent vocal fold palsy. ${ }^{17}$

In order to avoid or reduce thermal damage, this device has to be handled with care and should be positioned with the inactive blade turned toward the more delicate structures, with a lower power setting and with a shorter activation time. ${ }^{18}$

In addition, ultrasonic device, as any other electrocautery device, should be kept away from delicate anatomic structures, such as RLNs and the parathyroid glands.

In our study, the incidence of RLNs and parathyroid glands injuries was comparable in both groups. These results might be ascribed to the standardized surgical procedure used for both groups, which consists of identification and preservation of RLNs and of parathyroid glands. ${ }^{19-21}$ 
The lack of an increased number of RLNs or parathyroid glands injuries associated with the use of an ultrasonic device could be achieved not only with a correct use of the device but also with the combined help of magnification loupes.

Magnification $(2.5 \times$ or $3.5 \times)$ loupes are not exactly a proper surgical device, but can be considered a helpful support, as they enable a clearer vision of anatomy and enhance the precision of the surgical gesture. ${ }^{22}$

In this study, we highlighted that the correct preservation of critical neck structures is favored by the combination of an effective hemostasis performed by an ultrasonic device, since even minimal bleeding could blur the operative field, and greater visual acuity, guaranteed by magnification loupes.

Moreover, we found that the systematic use of the ultrasound scalpel with the support of magnification loupes is associated with a significant decrease of intraoperative bleeding and postoperative drainage. ${ }^{23}$ Besides, the decrease in drainage volumes leads to a reduction in the incidence of surgical site infection, which has been reported in none of our patients. ${ }^{24}$

The greater achievement with the simultaneous combination of magnification loupes and ultrasonic device is a significant reduction in operating time, as demonstrated in our study. It also avoids, to the patients, the discomfort of a long-duration general anesthesia with a potential faster recovery. ${ }^{25,26}$

Previous studies showed that the clamp-and-tie technique is more painful than the harmonic scalpel technique. As a matter of fact, the traditional technique relies on sutures, which are both foreign materials and can strangulate minor nerves that continue to produce action potentials, leading to pain immediately after surgery. Conversely, coagulated nerve produces no action potentials. The VAS score, which evaluated postoperative pain, was comparable in both our groups. This is probably due to the fact that total thyroidectomy is a surgical procedure that induces low levels of pain in general. ${ }^{27}$

A criticism of the use of the Harmonic FOCUS scalpel comes from its cost, as it is disposable and expensive. However, the price of the single-use device is counterbalanced by the considerable reduction in operative time, which in turn means performing more procedures in the same surgical session. ${ }^{23,25}$ The magnification loupes are reusable instead and we think they could be a valuable resource to any surgeon, not only in thyroid surgery.

\section{Conclusion}

In conclusion, our study showed that the combined use of magnification loupes and harmonic scalpel is correlated to an improvement of surgical outcomes and a significant reduction of operating time.

Indeed, the magnification of images enhances the surgeon's precision and enables a clearer vision of the anatomy, speeding up the operation significantly. A shorter operating time allows for optimal use of the operating room, leading to savings that in our experience can be quantified in about $12 \%$ of total expenditure, direct and indirect costs.

In addition, a reduction in surgical time avoids long-lasting anesthesia for the patient. Therefore, we both apply and recommend the use of the magnification loupes with the support of the ultrasound scalpel in thyroid surgery, in order to reduce operating times, work in a bloodless operating field, and aim to reduce postoperative complications.

\section{Clinical Significance}

Our results and suggestions can improve the standardized use of magnification loupes and harmonic scalpel in thyroid surgery.

\section{References}

1. Bellantone R, Lombardi CP, Bossola M, et al. Total thyroidectomy for management of benign thyroid disease: review of 526 cases. World J Surg 2002;26(12):1468-1471. DOI: 10.1007/s00268-002-6426-1.

2. Hannan SA. The magnificent seven: a history of modern thyroid surgery. Int J Surg 2006;4(3):187-191. DOI: 10.1016/j.ijsu.2006.03.002.

3. Rosato L, Avenia N, Bernante P, et al. Complications of thyroid surgery: analysis of a multicentric study on 14,934 patients operated in Italy over 5 years. World J Surg 2004;28(3):271-276. DOI: 10.1007/s00268003-6903-1.

4. Ravelli L, Damiani G, Bianchi CB, et al. Complications in thyroid surgery. Harmonic scalpel, harmonic focus vs conventional hemostasis: a meta-analysis. Int J Surg 2016;28(Suppl 1):22-32. DOI: 10.1016/j.ijsu.2015.12.050.

5. Kowalski LP, Sanabria A, Vartanian JG, et al. Total thyroidectomy with ultrasonic scalpel: a multicenter, randomized controlled trial. Head Neck 2012;34(6):805-812. DOI: 10.1002/hed.21815.

6. Ruggiero R, Gubitosi A, Conzo G, et al. Sutureless thyroidectomy. Int J Surg 2014;12(Suppl 1):189-193. DOI: 10.1016/j.ijsu.2014.05.011.

7. Geraci G, D'Orazio B, Chiarenza S, et al. Efficacy of fibrin sealant in thyroid surgery. Is drainage still necessary? Ann Ital Chir 2019;90:100105.

8. Bajwa MS, Tudur-Smith C, Shaw RJ, et al. Fibrin sealant in soft tissue surgery of the head and neck: a systematic review and meta analysis of randomized controlled trials. Clin Otolaryngol 2017;42(6):11411152. DOI: 10.1111/coa.12837.

9. Donohoe NO, Kintu-Luwaga R, Bolger J, et al. A prospective analysis of thyroidectomy outcomes in a resource limited setting. World J Surg 2015;39(7):1708-1711. DOI: 10.1007/s00268-015-3009-5.

10. Sista F, Schietroma M, Ruscitti C, et al. New ultrasonic dissector vs conventional hemostasis in thyroid surgery: a randomized prospective study. J Laparoendosc Adv Surg Tech A 2012;22(3):220224. DOI: $10.1089 /$ lap.2011.0266.

11. D'Ajello F, Cirocchi R, Docimo G, et al. Thyroidectomy with ultrasonic dissector: a multicentric experience. G Chir 2010;31(6-7):289-292.

12. Jiang $Y, G a o B$, Zhang $X$, et al. Prevention and treatment of recurrent laryngeal nerve injury in thyroid surgery. Int J Clin Exp Med 2014;7(1):101-107.

13. Carlander J, Koch C, Brudin L, et al. Heat production, nerve function and morphology following nerve close dissection with surgical instruments. World J Surg 2012;36(6):1361-1367. DOI: 10.1007/s00268012-1471-x.

14. Garas $\mathrm{G}$, Okabayashi $\mathrm{K}$, Ashrafian $\mathrm{H}$, et al. Which hemostatic device in thyroid surgery? A network meta-analysis of surgical technologies. Thyroid 2013;23(9):1138-1150. DOI: 10.1089/thy.2012.0588.

15. Lynch J, Parameswaran R. Management of unilateral recurrent laryngeal nerve injury after thyroid surgery: a review. Head Neck 2017;39(7):1470-1478. DOI: 10.1002/hed.24772.

16. Pisanu A, Porceddu G, Podda M, et al. Systematic review with metaanalysis of studies comparing intraoperative neuromonitoring of recurrent laryngeal nerves vs visualization alone during thyroidectomy. J Surg Res 2014;188(1):152-161. DOI: 10.1016/j. jss.2013.12.022.

17. Bakkar S, Papavramidis TS, Aljarrah Q, et al. Energy-based devices in thyroid surgery-an overview. Gland Surg 2020;9(Suppl 1):14-17. DOI: $10.21037 / \mathrm{gs} .2019 .08 .05$.

18. De Palma M, Rosato L, Zingone $F$, et al. Post thyroidectomy complications. The role of the devices: bipolar vs ultrasonic device. Collection of data from 1,846 consecutive patients undergoing thyroidectomy. Am J Surg 2016;212(1):116-121. DOI: 10.1016/j. amjsurg.2015.05.024. 
19. Mohebati A, Shaha AR. Anatomy of thyroid and parathyroid glands and neurovascular relations. Clin Anat 2012;25(1):19-31. DOI: 10.1002/ ca. 21220.

20. Pelizzo MR, Sorgato N, Merante Boschin I, et al. Does the ultrasound dissector improve parathyroid gland preservation during surgery? Eur J Surg Oncol 2014;40(7):865-868. DOI: 10.1016/j.ejso.2014. 03.007.

21. Fancy T, Gallagher 3rd D, Horning JD. Surgical anatomy of the thyroid and parathyroid glands. Otolaryngol Clin North Am 2010;43(2): 221-227. DOI: 10.1016/j.otc.2010.01.001.

22. Sapalidis K, Papanastasiou A, Fyntanidou V, et al. Comparison between magnification techniques and direct vision in thyroid surgery: a systematic review and meta-analysis. Medicina (Kaunas) 2019;55(11):725. DOI: 10.3390/medicina55110725.

23. Basurto-Kuba EOP, Robles-Estrada M, Hurtado-López LM, et al. Safety and cost-effectiveness in thyroidectomy using the HARMONIC scalpel compared to traditional hemostasis: a controlled clinical assay. Surg Technol Int 2017;30:141-147.

24. Moskalenko $M$, Asai M, Been $K$, et al. Incidence of surgical site infections after thyroid and parathyroid surgery: no role for antimicrobial prophylaxis. Am Surg 2018;84(6):897-901. DOI: 10.1177/000313481808400638.

25. Sebag F, Fortanier C, Ippolito G, et al. Harmonic scalpel in multinodular goiter surgery and cost analysis. J LaparoendosC Adv Surg Tech 2009;12(3):171-174. DOI: 10.1089/lap.2008.0043.

26. Luo $Y$, Li X, Dong J, et al. A comparison of surgical outcomes and complications between hemostatic devices for thyroid surgery: a network meta-analysis. Eur Arch Otorhinolaryngol 2017;274(3): 1269-1278. DOI: 10.1007/s00405-016-4190-3.

27. Papavramidis TS, Sapalidis K, Michalopoulos N, et al. Ultracision harmonic scalpel vs clamp-and-tie total thyroidectomy: a clinical trial. Head Neck 2010;32(6):723-727. 